\title{
Determination of Substellar Mass Function of Young Open Clusters Using 2MASS and GSC Data
}

\author{
Anandmayee Tej \\ Observatoire Astronomique de Strasbourg, 67000 Strasbourg, France
}

Kailash C. Sahu

Space Telescope Science Institute, Baltimore, MD 21218, USA

T. Chandrasekhar

Physical Research Laboratory, Navrangpura, Ahmedabad - 380009, India

N. M. Ashok

Physical Research Laboratory, Navrangpura, Ahmedabad - 380009, India

\begin{abstract}
We present a statistical method to derive the mass functions of open clusters using sky survey data such as the 2 Micron All Sky Survey (2MASS) and the Guide Star Catalogue (GSC). We have used this method to derive the mass functions in the stellar/substellar regime of three young, nearby open clusters, namely IC $348, \sigma$ Orionis and Pleiades. The mass function in the low mass range $\left(\mathrm{M}<0.50 M_{\odot}\right)$ is appreciably flatter than the stellar Salpeter function for all three open clusters. The contribution of objects below $0.5 \mathrm{M}_{\odot}$ to the total mass of the cluster is $\sim 40 \%$ and the contribution of objects below $0.08 \mathrm{M}_{\odot}$ to the total is $\sim 4 \%$.
\end{abstract}

\section{Introduction}

Recent surveys have found a significant population of low-mass stars, brown dwarfs and planetary-mass objects in young open clusters. Since low-mass objects evolve little over the lifetime of the Universe, the present day mass function of these objects is a good representation of the Initial Mass Function (IMF). The mass function in this low-mass regime is however poorly known due to faintness of these objects and also due to uncertainty in the mass-luminosity relations. Low-mass objects at or below the Hydrogen Burning Mass Limit (HBML) of $0.08 \mathrm{M}_{\odot}$ are known to be warmer and hence more luminous when young although they cool rapidly and fade with age (Baraffe et al. 1998). The combination of youth and proximity in some open clusters make them ideal targets for searches of low-mass objects below the HBML particularly at infrared wavelengths. In the present study we have adopted a statistical approach to determine the mass function $\left(\mathrm{dN} / \mathrm{dM} \propto \mathrm{M}^{-\alpha}\right)$ of objects in the mass range $0.5 M_{\odot}$ to $0.025-0.05 \mathrm{M}_{\odot}$ using data of three open clusters namely IC 348, $\sigma$ Orionis and Pleiades. 


\section{Sample Selection and Analysis}

We have used the data from two extended sky surveys - 2MASS Second Incremental Release and the latest version of GSC - on the three open clusters. The limiting magnitudes of $16.5,15.5,15$ and 21 in the $J, H, K$ and $F$ (POSS II IIIa-F) passbands, respectively, enable us to probe down to $0.025 \mathrm{M}_{\odot}$ in IC 348 and $\sigma$ Orionis and $0.04 \mathrm{M}_{\odot}$ in Pleiades. Unlike most other previous studies which rely on confirming candidate low-mass objects through spectroscopy we use a statistical approach to estimate the number of low-mass objects. In this approach it is important to use several control fields close to each cluster to subtract the contribution of foreground and background objects. The nature of these two extended surveys permitted us to use several control fields and variable field sizes. Table 1 lists the positions and radii of the fields chosen for the three clusters.

Table 1. Positions and sizes of the fields

\begin{tabular}{llcc}
\hline Fields & \multicolumn{2}{c}{ RA (J2000) } \\
& $\begin{array}{l}\text { Dec (J2000) } \\
\text { ( } \mathrm{h} \mathrm{s} \text { s }\end{array}$ & $\begin{array}{c}\text { Radius } \\
\text { (arcmin) }\end{array}$ \\
\hline IC 348 & 034430 & +321700 & 20 \\
Control 1 & 034908 & +311908 & 20 \\
Control 2 & 034410 & +331926 & 20 \\
& & & \\
$\sigma$ Orionis & 053845 & -023600 & 30 \\
Control 1 & 055829 & -042948 & 30 \\
Control 2 & 0.51100 & -002000 & 30 \\
& & & \\
Pleiades & 034700 & +240700 & 90 \\
Control 1 & 031800 & +264100 & 90 \\
Control 2 & 030500 & +244200 & 90 \\
\hline
\end{tabular}

In the first stage of analysis we merged the 2MASS and the GSC sources by taking 2MASS coordinates and cross correlating them with the GSC catalogue. A search radius of 2 "was found to be the optimum value for the cross correlation being small enough to reject spurious and multiple detections and large enough to include any minor positional uncertainties in the two catalogues. Selection criteria to pick out cluster members were derived by inspecting various colour magnitude diagrams (CMDs). The use of the F versus (F-R) CMD overcomes the degeneracies between the mass and distance found in the $\mathrm{K}_{s}$ versus $\left(\mathrm{J}-\mathrm{K}_{s}\right)$ $\mathrm{CMD}$, and minimizes the overlap between reddened background stars and lowmass members of the cluster. This leads to a more efficient rejection criteria for non members which is accomplished using the empirical data from Leggett (1992) and the theoretical isochrones of Baraffe et al. (1998). The selection criteria for low-mass members, derived using the suitably distance-scaled and extinction corrected model isochrones of Baraffe et al. (1998), are listed in Table 2. The sources satisfying the criteria are further statistically filtered for possible contaminants using the control fields. Details of the procedure are discussed in Tej et al. (2002). Finally, the masses of the selected candidates are determined 
by comparing observed magnitudes with those derived from evolutionary models (Baraffe et al. 1998 and Chabrier et al. 2000).

Table 2. Criteria for low-mass cluster members

\begin{tabular}{|c|c|c|}
\hline IC 348 & $\sigma$ Orionis & Pleiades \\
\hline $\begin{array}{c}F<[2.58(F-J) \\
+10.14]\end{array}$ & $\begin{array}{c}F<[2.55(F-J) \\
+10.26]\end{array}$ & $\begin{aligned} F< & {[7.93+1.99(F-J)} \\
& +056(F-J)^{2}-0.13(F-J)^{3} \\
& \left.+0.01(F-J)^{4}\right]\end{aligned}$ \\
\hline $\begin{array}{l}F-K \geq 4.09 \\
K \quad \geq 11.20 \\
J-K \geq 0.90 \\
H-K \geq 0.20\end{array}$ & $\begin{array}{l}F-K \geq 4.35 \\
K \quad \geq 11.17 \\
J-K \geq 0.94 \\
H-K \geq 0.20\end{array}$ & $\begin{array}{l}F-K \geq 3.42 \\
K \quad \geq 11.17 \\
J-K \geq 0.83 \\
H-K \geq 0.22\end{array}$ \\
\hline
\end{tabular}

\section{Results}

We first carried out a detailed study for IC 348. For this cluster, we derived the mass function using the solar metallicity models of Baraffe et al (1998) and dusty models of Chabrier et al. (2000) both of which gave similar results. The location of low-mass members isolated by us and the spectroscopically confirmed low-mass members of Luhman (1999) are in good agreement which builds the confidence in our selection criteria. We then used the same methodology for $\sigma$ Orionis and Pleiades. The resulting exponents of the mass functions for IC 348, $\sigma$ Orionis and Pleiades are respectively $0.7,1.2$ and 0.5 with an estimated error of \pm 0.2 . For the younger clusters IC 348 and $\sigma$ Orionis, there is some hint of a possible dip in the mass function at the position of the HBML.

Table 3. Summary of the results

\begin{tabular}{lllll}
\hline Cluster & $\begin{array}{l}\text { Age } \\
(\mathrm{Myr})\end{array}$ & $\begin{array}{l}\text { Distance } \\
(\mathrm{pc})\end{array}$ & $\begin{array}{l}\text { Mass Range } \\
\left(\mathrm{M}_{\odot}\right)\end{array}$ & $\alpha$ \\
\hline IC 348 & 5 & 316 & $0.5-0.035$ & 0.7 \\
$\sigma$ Orionis & 3 & 352 & $0.5-0.045$ & 1.2 \\
Pleiades & 100 & 125 & $0.5-0.055$ & 0.5 \\
\hline
\end{tabular}

The results of this statistical approach (Table 3 ) imply that though the mass function continues to rise above the HBML it is appreciably flatter compared to the Salpeter mass function. In Figure 1 we have plotted the derived mass function for the three clusters. Taking the canonical Salpeter exponent of 2.35 in the mass range $1-10 \mathrm{M}_{\odot}$, the Chabrier exponent of 1.55 in the mass range $0.5-1.0 \mathrm{M}_{\odot}$ and the values obtained by us below $0.5 \mathrm{M}_{\odot}$ we estimate the mass contribution to be about $40 \%$ for objects below $0.5 \mathrm{M}_{\odot}$ and $4 \%$ for objects below the HBML of $0.08 \mathrm{M}_{\odot}$. Our results are consistent with previous studies (e.g. Béjar et al., 2001) and suggest that although low-mass objects are at least as numerous as their stellar counterparts their contribution to the total mass of the cluster is small. 

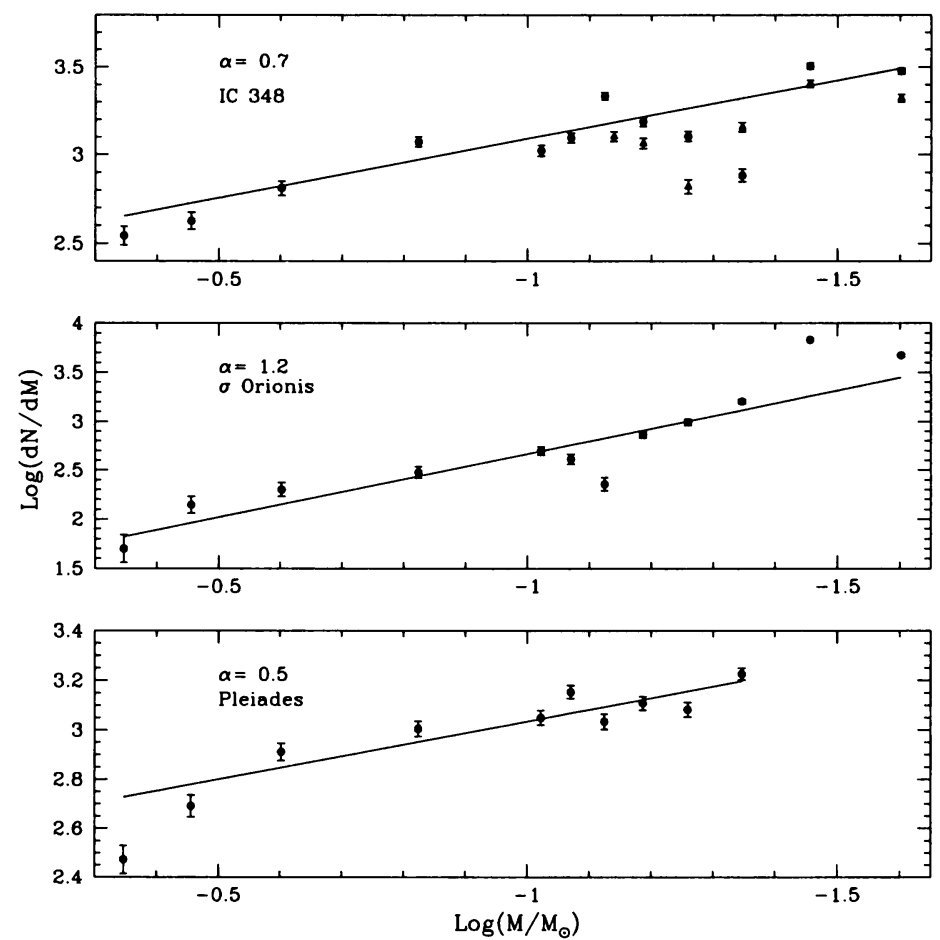

Figure 1. The derived mass functions for the three clusters. The yerror bars are the $\sqrt{N}$ errors involved in the counting statistics. For all three clusters the filled circles are points derived using the models of Baraffe et al. (1998) and for IC 348 the open triangles are data points derived using the dusty isochrones of Chabrier et al. (2000).

Acknowledgments. We would like to thank Brian McLean and Mario Lattanzi (GSC-II project scientists) for access to the development version of GSC-II in advance of publication. We are grateful to I. Baraffe and F. Allard for making electronic versions of the latest models available and generating model isochrones for the nonstandard $\mathrm{F}$ passbands.

\section{References}

Baraffe, I., Chabrier, G., Allard,F., \& Hauschildt, P.H. 1998, A\&A, 337, 403

Béjar, V.J.S., Martín, E.L., Zapatero Osorio, M.R., et al. 2001, ApJ, 556, 830

Chabrier, G., Baraffe, I., Allard, F., \& Hauschildt, P.H. 2000, ApJ, 542, 464

Leggett, S.K. 1992, ApJS, 82, 351

Luhman, K. L. 1999, ApJ, 525, 466

Tej A., Sahu, K.C., Chandrasekhar, T. and Ashok, N.M. 2002 ApJ (in press) (astro-ph/0206325) 